\title{
AUTOSOMAL RECESSIVE HYPER IgM SYNDROME ASSOCIATED WITH ACTIVATION-INDUCED CYTIDINE DEAMINASE GENE IN THREE TURKISH SIBLINGS PRESENTED WITH TUBERCULOSIS LYMPHADENITIS - CASE REPORT
}

\author{
TURKAN PATIROGLU ${ }^{1,3}$, H. HALUK AKAR ${ }^{1 *}$, \\ MIRJAM VAN DER BURG ${ }^{2}$ and EKREM UNAL ${ }^{3}$ \\ ${ }^{1}$ Erciyes University School of Medicine, Department of Pediatric Immunology, \\ Kayseri, Turkey \\ ${ }^{2}$ Department of Erasmus MC, University Medical Center Rotterdam, \\ Rotterdam, the Netherlands \\ ${ }^{3}$ Erciyes University School of Medicine, Department of Pediatric Hematology and Oncology, \\ Kayseri, Turkey
}

(Received: 2 February 2015; accepted: 4 April 2015)

The hyper-immunoglobulin M (HIGM) syndrome is a heterogeneous group of genetic disorders characterized by recurrent infections, decreased serum levels of immunoglobulin $\mathrm{G}(\mathrm{IgG})$ and $\mathrm{IgA}$, and normal/increased serum levels of IgM. Herein, we describe three Turkish siblings with HIGM syndrome who had a homozygous missense mutation (c.70C $>$ T, p.Arg24Trp) in the activation-induced cytidine deaminase gene which results in autosomal recessive HIGM syndrome. Two of the siblings, sibling 1 and sibling 3, presented with cervical deep abscess and cervical tuberculosis lymphadenitis, respectively.

Keywords: hyper-immunoglobulin $\mathrm{M}$ syndrome, activation-induced cytidine deaminase gene, tuberculosis lymphadenitis

\section{Introduction}

Patients with hyper-immunoglobulin M (HIGM) syndrome incline to recurrent infections, lymphoid hyperplasia, autoimmune, and inflammatory disorders. This syndrome can be inherited as X-linked, autosomal recessive (AR), or occasionally dominant mutations (AD). The main defect results from mutations

*Corresponding author; E-mail: himmetakar@gmail.com 
in the B-cell signaling genes, which are required for class switch recombination (CSR) and somatic hypermutation (SHM) [1]. At least six molecular defects associated with $\mathrm{X}$-linked (CD40 ligand and the nuclear factor $\kappa \mathrm{B}$ essential modulator (NEMO genes), AR [CD40, activation-induced cytidine deaminase (AID) / or as known activation-induced cytidine deaminase $(A I C D A)$, uracil-DNA glycosylase $(U N G)$ genes], and AD [inhibitor of NF-B $(I \kappa B \alpha)$ gene] have been reported as causing HIGM syndrome [2]. The AID gene mutations are the most frequent causative aberration in the AR HIGM patients. Defective gene product, AID protein, leads to the impairment of CSR and SHM generation [3-4]. More than 40 different mutations have been identified in the AID gene in patients with HIGM syndrome [5]. Herein, we report three Turkish siblings who had a homozygous missense mutation in AID gene. One of them, sibling 1, presented with cervical deep abscess. The other one, sibling 3, presented with cervical tuberculosis lymphadenitis.

\section{Clinical Reports}

In this study, we describe three siblings (two males, one female). There was second degree consanguinity between parents. The laboratory findings (elevated IgM, decreased IgG and IgA) were consistent with HIGM syndrome. A homozygous missense mutation (c.70C $>\mathrm{T}$, p.Arg24Trp) was found in exon 2 in the $A I D$ gene. After the genetic diagnosis, hematopoietic stem cell transplantation (HSCT) was planned for the patients (Table I).

\section{Sibling 1}

An 11-year-old girl was admitted with left cervical mass to the Department of Pediatric Emergency Unit. Physical examination revealed $3 \times 3 \mathrm{~cm}$ fluctuated, hyperemic, mobile, and painful mass in left sternocleidomastoid muscle (SCM) posterolateral region. Also, she had cervical, bilateral inguinal multiple millimetric mobile lymphadenopathies, and enlarged tonsils. Her growth parameters were normal. Cervical ultrasound showed $14 \times 40 \times 42 \mathrm{~mm}$ lobulated mass which is consistent with abscess in left supraclavicular area and multiple cervical lymphadenopathies (Fig. 1). Staphylococcus aureus was cultured from the drained abscess material. Tbc was investigated because of her brother experienced Tbc lymphadenitis. The results of draining materials for tuberculosis were negative. No acidfast bacilli were seen nor cultured, Tbc polymerase chain reaction (PCR) and PPD were negative. Clinical improvement was observed with cefazolin and IVIG 


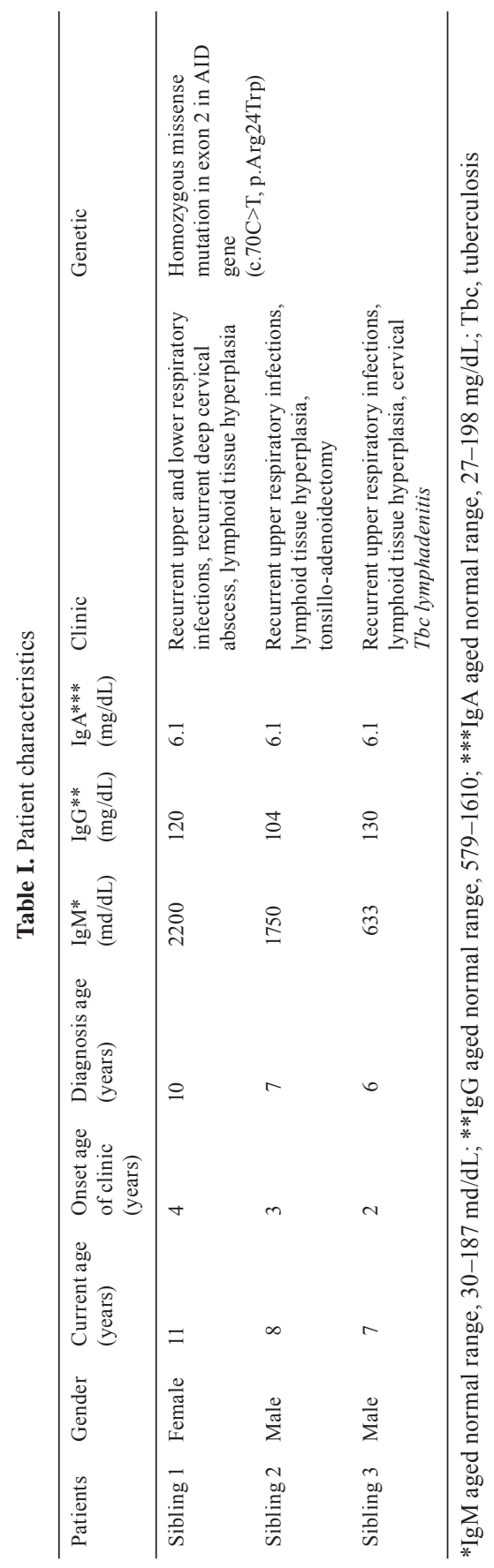




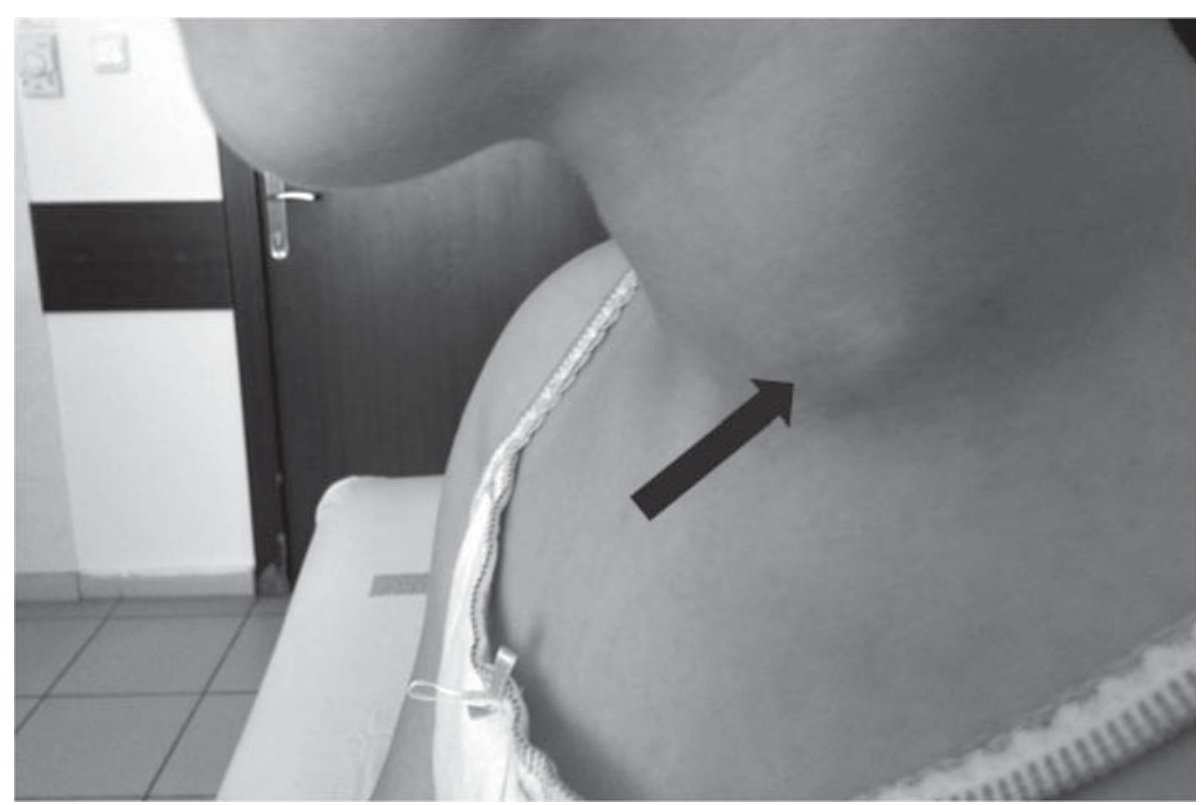

Figure 1. Cervical deep abscess in patient 1

therapy in two weeks. It was learned from the anamnesis, her medical problems started at 4 years of age with recurrent otitis, sinusitis, and pneumonia. She was treated lots of antibiotics cures. She was interned three times due to pneumonia. In her last two years of life, she experienced two times recurrent cervical painful mass which was spontaneously draining as in presented to our clinic. After genetic diagnosis AR HIGM syndrome, monthly IVIG infusion and the trimethoprim-sulfamethoxazole (TMP-SMX) prophylaxis for Pneumocystis jiroveci pneumonia (PcP) was stared.

\section{Sibling 2}

Second sibling was an 8-year-old boy. His problems started at 3 years of age with recurrent upper respiratory infections such as otitis media and sinusitis. He underwent tonsillo-adenoidectomy when he was 5 years old due to recurrent otitis media. Also he had frequent courses of treatments with antibiotics as in other siblings. The same prophylaxis regimen (monthly IVIG and TMP-SMX for PcP) was initiated as in sibling 1. 


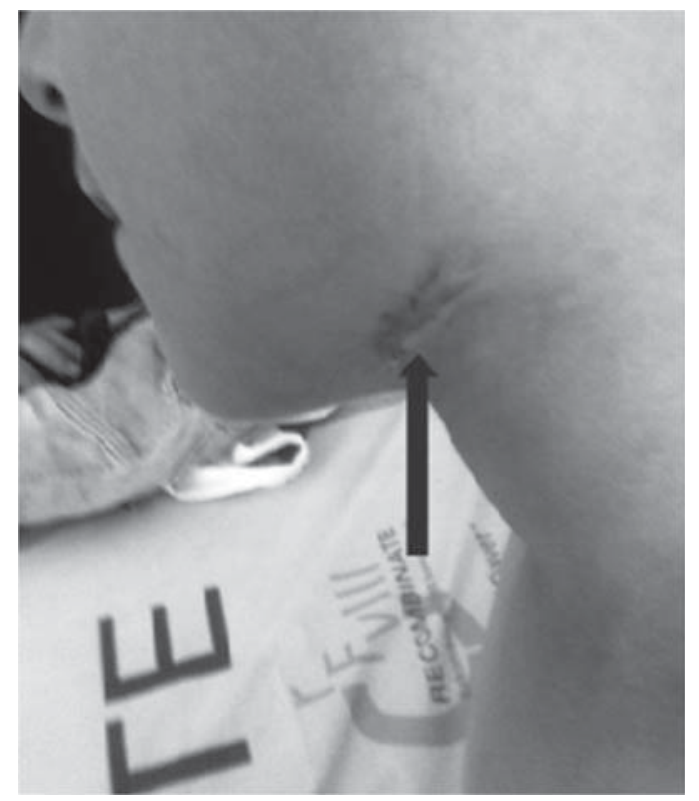

Figure 2. Sequelae of tuberculous lymphadenitis in patient 3

Sibling 3

A 7-year-old boy was referred to the Department of Pediatric Immunology because of further diagnostic work-up with the other siblings. His medical problems started at 2 years of age with recurrent upper respiratory tract infections. At the age of 5 years, he was admitted to hospital with cervical mass. Physical examination revealed an anterior cervical $3 \times 2 \mathrm{~cm}$ fluctuated, painful mass and cervical multiple millimetric lymphadenopathies. Cervical ultrasound showed $34 \times 18 \mathrm{~mm}$ lobulated heterogenic abscess formation mass in left submandibular area and $20 \times 17 \times 30 \mathrm{~mm}$ in view of the heterogeneous solid mass (conglomerated lymphadenopathy) in anterior left SCM region and multiple millimetric cervical lymphadenopathies. Histopathologic examination showed chronic non-caseating granulomatous inflammation in the cervical mass biopsy. Acid-fast bacilli were seen. Also, Tbc was cultured from the biopsy material with BACTEC automated system. It was learned that the family lives with the grandfather who experienced lung Tbc disease 5 years ago. He had been treated with anti-tuberculosis treatment for one year. Clinical improvement was seen with triple antituberculosis treatment in one year (Fig. 2). Also, the same prophylaxis regimen (monthly IVIG and TMP-SMX for PcP) was stared to the patient. 


\section{Discussion}

$\mathrm{X}$-linked HIGM syndrome is the most common form of HIGM, which is caused by mutations in CD4OL and NEMO genes, presents recurrent infections such as PcP and Cryptosporidium infections [6]. The AID gene encodes AID protein consists of 198 amino acids. In SHM and CSR, the role of AID gene has not yet been clearly elucidated. For this, in the medical literature, two molecular mechanisms have been presumed as DNA deamination and RNA-editing hypotesis [7-9]. Patients with AR HIGM syndrome usually have normal B lymphocyte counts as in the presented patients. However, in contrast to the $\mathrm{X}$ linked patients, B cells are not able to switch from IgM secreting cells to the $\operatorname{IgG}, \operatorname{IgA}$, and IgE secreting cells even though CD40 antibody in the patients with AR HIGM syndrome. Namely in the patients with AR HIGM syndrome, there is a really intrinsic B cell abnormality. Patients with AR HIGM syndrome usually have lymphoid hyperplasia, recurrent respiratory and gastrointestinal infections with bacterial agents. These patients usually do not have susceptibility to opportunistic infections such as PcP. Also, these patients less likely have neutropenia according to the X-linked patients with HIGM syndrome [10-11]. Neutropenia was not observed in the presented siblings. The AR HIGM syndrome results from the $A I D$ gene mutations might be complicated autoimmune and inflammatory disorders including polyarthritis, autoimmune hepatitis, hemolytic anemia, immune thrombocytopenia [12]. In the presented patients, autoimmune manifestation was not observed up to date. In patients with AR HIGM syndrome, also the diagnosis of HIGM may be delayed to after first decade of life as in sibling 1. The high levels of IgM antibodies ensure effective protection against some infectious agents in the patients with AR HIGM syndrome [5]. Thus, infections are not enough frequent and severe as in the presented patients to reveal a suspicion of immunodeficiency disease at early ages [10]. Likewise in the all presented siblings, the diagnosis of the AR HIGM syndrome was identified after the age of 5 years. Patients with X-linked HIGM syndrome often present opportunistic infections such as PcP, Cryptosporidium species, Toxoplasma gondii, and Mycobacteria species. Almost $40 \%$ of opportunistic infections results from PcP in the patients with HIGM syndrome [13]. Tbc has been reported as 9-28\% in the patients with $\mathrm{X}$ linked HIGM syndrome [14-15]. In contrast to the patients with $\mathrm{X}$ linked HIGM syndrome, the patients with AR HIGM syndrome caused by mutations in AID gene do not have opportunistic infections. It has been explained with AR HIGM syndrome is a pure B-cell deficiency disease [5]. In the medical literature, to our knowledge, Tbc infections have been reported in only one patient with AR HIGM as Tbc osteomyelitis [16]. In our patients, sibling 3 presented with Tbc lymphadenitis. 
As mentioned above, recurrent infections are mainly bacterial and mostly are observed as pneumonia in the $A I D$ gene deficient patients. In these patients, other sites of infection are the skin, lymph nodes, gastrointestinal tract, and central nervous system such as meningitis and encephalitis [3, 12]. In the medical literature, also, infections as abscess formation have been reported in only Xlinked HIGM patients [11, 17]. To our knowledge, deep abscess have been reported rarely in the patients with AR HIGM syndrome [16]. Sibling 1 presented with recurrent cervical deep abscess.

In conclusion, AID gene deficient patients have an increased risk of recurrent, severe infections leading to chronic sinopulmonary infections such as sinusitis, bronchiectasis, and lymphoid hyperplasia. Also, autoimmune and inflammatory disorders can be observed in the follow up of patients with AR HIGM syndrome. In addition to these informations, in the presented sibling 1 and 3, recurrent deep cervical abscess and cervical Tbc lymphadenitis were observed, respectively.

\section{Conflict of Interest}

The authors declare that they have no conflict of interest.

\section{References}

1. Stavnezer, J., Guikema, J. E., Schrader, C. E.: Mechanism and regulation of class switch recombination. Annu Rev Immunol 26, 261-292 (2008).

2. Lee, W. I., Huang, J. L., Ye, K. W., Yang, M. J., Lai, M. C., Chen, L. C., Ou, L. S., Yao, T. C., Lin, S. J., Jaing, T. H., Chen, S. H., Hsieh, M. Y., Yu, H. H., Chien, Y. H., Shyur, S. D.: Clinical features and genetic analysis of Taiwanese patients with the hyper IgM syndrome phenotype. Pediatr Infect Dis J 32, 1010-1016 (2013).

3. Davies, E. G., Thrasher, A. J.: Update on the hyper immunoglobulin M syndromes. Br J Haematol 149, 167-180 (2010).

4. Erdos, M., Durandy, A., Marodi, L.: Genetically acquired class-switch recombination defects: The multi-faced hyper-IgM syndrome. Immunol Lett 97, 1-6 (2005).

5. Mahdaviani, S. A., Hirbod-Mobarakeh, A., Wang, N., Aghamohammadi, A., Hammarström, L., Masjedi, M. R., Pan-Hammarström, Q., Rezaei, N.: Novel mutation of the activation-induced cytidine deaminase gene in a Tajik family: Special review on hyperimmunoglobulin M syndrome. Expert Rev Clin Immunol 8, 539-546 (2012).

6. Winkelstein, J. A., Marino, M. C., Ochs, H., Fuleihan, R., Scholl, P. R., Geha, R., Stiehm, E. R., Conley, M. E.: The X-linked hyper-IgM syndrome: Clinical and immunologic features of 79 patients. Medicine 82, 373-384 (2003).

7. Shinkura, R., Tian, M., Smith, M., Chua, K., Fujiwara, Y., Alt, F. W.: The influence of transcriptional orientation on endogenous switch region function. Nat Immunol 4, 435441 (2003). 
8. Shivarov, V., Shinkura, R., Doi, T., Begum, N. A., Nagaoka, H., Okazaki, I. M., Ito, S., Nonaka, T., Kinoshita, K., Honjo, T.: Molecular mechanism for generation of antibody memory. Philos Trans R Soc Lond B Biol Sci 364, 569-567 (2009).

9. Xu, Z., Pone, E. J., Al-Qahtani, A., Park, S. R., Zan, H., Casali, P.: Regulation of aicda expression and AID activity: Relevance to somatic hypermutation and class switch DNA recombination. Crit Rev Immunol 27, 367-397 (2007).

10. Minegishi, Y., Lavoie, A., Cunningham-Rundles, C., Bedard, P. M., Hebert, J., Cote, L., Dan, K., Sedlak, D., Buckley, R. H., Fischer, A., Durandy, A., Conley, M. E.: Mutations in activation-induced cytidine deaminase in patients with hyper IgM syndrome. Clin Immunol 97, 203-210 (2000).

11. Shah, I., Rahangdale, A., Bhatnagar, S.: Liver abscesses and hyper IgM syndrome. J Family Med Prim Care 2, 206-208 (2013).

12. Quartier, P., Bustamante, J., Sanal, O., Plebani, A., Debré, M., Deville, A., Litzman, J., Levy, J., Fermand, J. P., Lane, P., Horneff, G., Aksu, G., Yalçin, I., Davies, G., Tezcan, I., Ersoy, F., Catalan, N., Imai, K., Fischer, A., Durandy, A.: Clinical, immunologic and genetic analysis of 29 patients with autosomal recessive hyper-IgM syndrome due to Activation-Induced Cytidine Deaminase deficiency. Clin Immunol 110, 22-29 (2004).

13. Montella, S., Maglione, M., Giardino, G., Di Giorgio, A., Palamaro, L., Mirra, V., Ursini, M. V., Salerno, M., Pignata, C., Caffarelli, C., Santamaria, F.: Hyper IgM syndrome presenting as chronic suppurative lung disease. Ital J Pediatr 38, 45 (2012).

14. Madkaikar, M., Gupta, M., Chavan, S., Italia, K., Desai, M., Merchant, R., Radhakrishnan, N., Ghosh, K.: X-linked hyper IgM syndrome: Clinical, immunological and molecular features in patients from India. Blood Cells Mol Dis 53, 99-104 (2014).

15. Cabral-Marques, O., Klaver, S., Schimke, L. F., Ascendino, E. H., Khan, T. A., Pereira, P. V., Falcai, A., Vargas-Hernández, A., Santos-Argumedo, L., Bezrodnik, L., Moreira, I., Seminario, G., Di Giovanni, D., Raccio, A. G., Porras, O., Weber, C. W., Ferreira, J. F., Tavares, F. S., de Carvalho, E., Valente, C. F., Kuntze, G., Galicchio, M., King, A., Rosário-Filho, N. A., Grota, M. B., dos Santos Vilela, M. M., Di Gesu, R. S., Lima, S., de Souza Moura, L., Talesnik, E., Mansour, E., Roxo-Junior, P., Aldave, J. C., Goudouris, E., Pinto-Mariz, F., Berrón-Ruiz, L., Staines-Boone, T., Calderón, W. O., del Carmen ZarateHernández, M., Grumach, A. S., Sorensen, R., Durandy, A., Torgerson, T. R., Carvalho, B. T., Espinosa-Rosales, F., Ochs, H. D., Condino-Neto, A.: First report of the hyper-IgM syndrome registry of the Latin American Society for Immunodeficiencies: Novel mutations, unique infections, and outcomes. J Clin Immunol 34, 146-156 (2014).

16. Shah, I.: Hyper IgM syndrome with tuberculous osteomyelitis and scrofuloderma. Indian Pediatr 42, 952-953 (2005).

17. Tsai, H. Y., Yu, H. H., Chien, Y. H., Chu, K. H., Lau, Y. L., Lee, J. H., Wang, L. C., Chiang, B. L., Yang, Y. H.: X-linked hyper-IgM syndrome with CD40LG mutation: Two case reports and literature review in Taiwanese patients. J Microbiol Immunol Infect 48, 113-118 (2015). 